\title{
Effect of dexmedetomidine anesthesia on perioperative levels of TNF- $\alpha$ and IL-6 in patients with ovarian cancer
}

\author{
MAODONG LIU ${ }^{1}$, YUSHENG $\mathrm{YI}^{2}$ and MINGQIANG ZHAO \\ Departments of ${ }^{1}$ Anesthesiology and ${ }^{2}$ Pain Management, The Affiliated Hospital of Qingdao University; \\ ${ }^{3}$ Department of Anesthesiology, Qingdao Center Hospital, Qingdao, Shandong 266000, P.R. China
}

Received August 27, 2018; Accepted April 8, 2019

DOI: 10.3892/ol.2019.10247

\begin{abstract}
Effect of continuous use of dexmedetomidine during general anesthesia on perioperative levels of tumor necrosis factor- $\alpha$ (TNF- $\alpha$ ) and interleukin-6 (IL-6) in patients undergoing radical resection of ovarian cancer was investigated. The initial treatment of ovarian cancer is mainly radical surgery. Most patients with ovarian cancer radical surgery can achieve good results, but the use of improper anesthetic drugs in radical surgery can easily lead to unstable patient vital signs. Therefore, the selection of appropriate anesthetic drugs has become the key to radical ovarian cancer surgery. There are few reports on the use of dexmedetomidine in anesthesia for ovarian cancer radical surgery. This study was performed to retrospectively analyze the case data of patients undergoing laparoscopic ovarian cancer radical surgery, and to compare the hemodynamics of dexmedetomidine anesthesia with midazolam anesthesia and the concentrations of TNF- $\alpha$ and IL-6, to provide reference for clinical implementation of ovarian cancer radical surgery. The hemodynamics of patients in the dexmedetomidine group were stable compared with the midazolam group. Serum TNF- $\alpha$ and IL-6 levels were significantly lower in the dexmedetomidine group than that in the midazolam group. If dexmedetomidine were continuously used during general anesthesia, the perioperative serum levels of TNF- $\alpha$ and IL- 6 could be effectively reduced in patients undergoing radical resection of ovarian cancer, and the perioperative stress response was suppressed.
\end{abstract}

\section{Introduction}

Ovarian cancer is a malignancy of the female genital systems. The incidence of ovarian cancer ranks third in gynecologic malignancies, second only to cervical cancer and endometrial

Correspondence to: Dr Mingqiang Zhao, Department of Anesthesiology, Qingdao Center Hospital, 127 Siliu South Road, Qingdao, Shandong 266000, P.R. China

E-mail: nps2x7@163.com

Key words: laparoscopic radical resection of ovarian cancer, dexmedetomidine, general anesthesia, TNF- $\alpha$, IL- 6 , midazolam cancer. However, its mortality rate ranks first in gynecologic malignancies (1). The diagnostic rate of ovarian cancer is relatively low due to imaging limitations for early diagnosis and the possibility of multifocal origin (2). There are no obvious and reliable clinical symptoms in the early stage of ovarian cancer. Thus, missed diagnosis and misdiagnosis can occur. Often when clinical symptoms appear, the cancer cells have already spread to the abdominal organs. Approximately $65 \%$ of patients diagnosed with ovarian cancer are at advanced stage at the time of diagnosis (3). The primary treatment of ovarian cancer is radical resection. Good outcomes can be achieved for most patients undergoing radical resection of ovarian cancer. However, improper use of anesthetic drugs in ovarian cancer surgery can cause unstable vital signs and even lead to respiratory depression and other adverse reactions (4). Therefore, selection of appropriate anesthetic drugs has become the key to the success of ovarian cancer radical surgery.

Midazolam is extensively used in clinic as the preferred anesthesia for radical resection of ovarian cancer due to fast onset of action and rapid metabolism. However, it was reported that multiple postoperative adverse reactions, such as delirium, hallucination, hypotension, and even thrombophlebitis, can occur following the use of midazolam anesthesia (5). Dexmedetomidine is a novel receptor agonist, which causes less adverse reactions and has no respiratory depression effect (6). According to literature, under general anesthesia, single dose of $0.5 \mu \mathrm{g} / \mathrm{kg}$ dexmedetomidine given before extubation can attenuate extubation reflexes, stabilizing patient hemodynamics and vital signs (7). Tumor necrosis factor $\alpha$ (TNF- $\alpha$ ) is an important cytokine. An appropriate amount of TNF- $\alpha$ can kill target cells and regulate adaptive immunity, so as to protect the body. Interleukin-6 (IL-6) is a cytokine involved in the body's inflammatory response. In some types of cancer, IL-6 is secreted as a growth factor to stimulate tumor growth.

The function of the ovaries is to produce steroid hormones and egg cells to maintain female reproductive function and endocrine homeostasis. It is an important reproductive organ in women (8). In recent years, ovarian tumors have become one of the common tumors affecting the quality of life of women (9). The mortality rate of ovarian cancer ranks first in gynecological tumors. Therefore, it is of great significance to improve the diagnosis and treatment of ovarian malignant tumors (10). There is no obvious symptom in the early stage of ovarian cancer. When the pelvic tumor spreads to the 
Table I. Baseline clinical data of 343 patients undergoing laparoscopic radical resection of ovarian cancer (n, \%).

\begin{tabular}{|c|c|c|c|c|}
\hline Variable & Dexmedetomidine group $(\mathrm{n}=169)$ & Midazolam group $(\mathrm{n}=174)$ & $\chi^{2}$ & P-value \\
\hline \multicolumn{5}{|l|}{ Age } \\
\hline$<40$ & $74(43.79)$ & $81(46.55)$ & \multirow[t]{2}{*}{0.265} & \multirow[t]{2}{*}{0.607} \\
\hline$\geq 40$ & $95(56.21)$ & $93(53.45)$ & & \\
\hline \multicolumn{5}{|l|}{ Marital status } \\
\hline Yes & $141(83.43)$ & $149(85.63)$ & \multirow[t]{2}{*}{0.318} & \multirow[t]{2}{*}{0.573} \\
\hline No & $28(16.57)$ & $25(14.37)$ & & \\
\hline \multicolumn{5}{|l|}{ Pregnancy } \\
\hline Yes & $115(68.05)$ & $113(64.94)$ & \multirow[t]{2}{*}{0.371} & \multirow[t]{2}{*}{0.543} \\
\hline No & $54(31.95)$ & $61(35.06)$ & & \\
\hline \multicolumn{5}{|l|}{ Peritoneal effusion } \\
\hline Yes & $135(79.88)$ & $132(75.86)$ & \multirow[t]{2}{*}{0.803} & \multirow[t]{2}{*}{0.37} \\
\hline No & $34(20.12)$ & $42(24.14)$ & & \\
\hline \multicolumn{5}{|l|}{ Pathological classification } \\
\hline Epithelial ovarian cancer & $152(89.94)$ & $155(89.08)$ & \multirow[t]{3}{*}{1.901} & \multirow[t]{3}{*}{0.387} \\
\hline Ovarian sex cord-stromal tumors & $11(6.51)$ & $8(4.60)$ & & \\
\hline Others & $6(3.55)$ & $11(6.32)$ & & \\
\hline \multicolumn{5}{|l|}{ FIGO stage } \\
\hline Stage I & $21(12.43)$ & $26(14.94)$ & \multirow[t]{4}{*}{2.144} & \multirow[t]{4}{*}{0.543} \\
\hline Stage II & $87(51.48)$ & $76(43.68)$ & & \\
\hline Stage III & $54(31.95)$ & $63(36.21)$ & & \\
\hline Stage IV & 7 (4.14) & $9(5.17)$ & & \\
\hline
\end{tabular}

abdominal cavity, there may be signs such as abdominal distension, abdominal pain and bulging sensation, which may be accompanied by irregular bleeding or irregular menstruation. The symptoms are not typical and are easily misdiagnosed or fail-diagnosed $(11,12)$. It has been reported (13) that if ovarian cancer is effectively treated before it is transferred, the survival rate of patients can be increased to up to $90 \%$, so early diagnosis and early treatment are important for patients with ovarian cancer. The operation with fertility preservation has low safety and the recurrence rate is high, therefore, most patients with stage II or above undergo radical ovarian surgery (14).

Dexmedetomidine anesthesia use has rarely been reported in radical resection of ovarian cancer. In this study, the clinical records of patients undergoing laparoscopic radical resection of ovarian cancer were retrospectively analyzed. The hemodynamics, as well as the serum levels of TNF- $\alpha$ and IL-6, was compared between patients treated with dexmedetomidine anesthesia and midazolam anesthesia, in order to provide a reference for the clinical procedures of ovarian cancer radical surgery.

\section{Patients and methods}

Clinical data. A total of 343 patients with ovarian cancer who underwent radical resection from April 2013 to June 2016 in the Affiliated Hospital of Qingdao University (Qingdao, China) were selected for retrospective analysis. Among the patients, 169 were treated with dexmedetomidine (dexmedetomidine group). The patient age in this group ranged from 21 to 74 years, with an average age of $36.62 \pm 4.15$ years. The remaining 174 patients were treated with midazolam (midazolam group). The patient age in this group ranged from 19 to 71 years, with an average age of $38.35 \pm 5.32$ years. In order to reach accurate and reliable results, the baseline clinical data of patients in the two groups were compared, and it was found that all the variables were comparable $(\mathrm{P}>0.05)$. The baseline clinical data are listed in Table I.

The study was approved by the Ethics Committee of the Affiliated Hospital of Qingdao University (Qingdao, China).

Inclusion and exclusion criteria. Patients who met the following criteria were eligible for the study: i) Patients who were diagnosed with ovarian cancer by pathological biopsy in the above hospital; ii) patients who underwent laparoscopic radical resection of ovarian cancer in the hospital after diagnosis; iii) patients who received total intravenous anesthesia; iv) patients who had complete clinical records; and v) patients aged $>18$ years; patients with ASA physical status score 2 or 3 . Patients who met the following criteria were excluded from the study: i) Patients who were allergic to the drugs used in this study; ii) patients who were pregnant or in lactation; iii) patients who had acute gastrointestinal hemorrhage or other serious diseases; and iv) patients who had communication disorders or cognitive disorders. The patients and their families signed an informed consent and cooperated with medical staff to complete the study.

Methods. The patients underwent $8 \mathrm{~h}$ fasting and $4 \mathrm{~h}$ waterdeprivation before surgery. After entering the operating room, 
the patient was connected to a ProSim 8 vital signs simulator (Fluke Electronics Corp., Everett, WA, USA) for monitoring the electrocardiogram and oxygen saturation. General anesthesia was induced using $2-3 \mathrm{mg} / \mathrm{kg}$ of fentanyl, $1.5 \mathrm{mg} / \mathrm{kg}$ of propofol, $0.05 \mathrm{mg} / \mathrm{kg}$ of midazolam, and $0.12 \mathrm{mg} / \mathrm{kg}$ of vecuronium bromide through i.v. injection. The pump infusion rate and dosage were adjusted based on the patient's heart rate (HR) and blood pressure. After that, the nasal catheter was used to take oxygen at the speed of $41 / \mathrm{min}$ until the end of the operation. A venous blood sample was drawn before oxygen inhalation (T1), shortly after extubation (T2), and on the morning of day 1 (T3) and day 2 (T4) after surgery, respectively (15). The serum levels of TNF- $\alpha$ and IL-6 (primary endpoint) were measured by enzyme-linked immunosorbent assay (ELISA) using a kit manufactured by Wuhan Sanying Biotech Inc. (Wuhan, China) with a lot no. 5200103007. The mean arterial pressure (MAP) and heart rate (HR) (secondary endpoint) were recorded at each time point.

Statistical analysis. Statistical analysis was performed using SPSS 19.1 (IBM Corp., Armonk, NY, USA) software system. Enumeration data were expressed in percentage, and the $\chi^{2}$ test was used to compare differences between the two groups. Measurement data were expressed as mean $\pm \mathrm{SD}$, and the t-test was used to compare differences between the two groups. A difference was statistically significant at $\mathrm{P}<0.05$.

\section{Results}

MAPs of patients in the dexmedetomidine group and the midazolam group at different time points. At T1, T2, T3 and T4, the MAPs of patients in the dexmedetomidine group were (76.83 \pm 7.17$) \mathrm{mmHg},(77.91 \pm 8.14) \mathrm{mmHg},(78.19 \pm 7.83) \mathrm{mmHg}$, and $(77.96 \pm 8.21) \mathrm{mmHg}$, respectively, while the MAPs of patients in the midazolam group were $(75.27 \pm 8.42) \mathrm{mmHg}$, $(83.63 \pm 6.92) \mathrm{mmHg},(87.71 \pm 7.16) \mathrm{mmHg}$, and $(80.57 \pm 8.47)$ $\mathrm{mmHg}$, respectively (Fig. 1). The MAPs at T1 in both groups were comparable, and there was no significant difference between them $(\mathrm{P}>0.05)$. However, the MAPs at T2, T3 and T4 were all lower in the dexmedetomidine group than those in the midazolam group, and the differences were statistically significant $(\mathrm{P}<0.05)$. Within the dexmedetomidine group, the MAPs at each time point were comparable, and the differences were not statistically significant $(\mathrm{P}>0.05)$. Within the midazolam group, the MAPs at T2 and T3 were higher than that at the preceding time point, whereas the MAP at T4 was lower than that at the preceding time point. All the differences were statistically significant $(\mathrm{P}<0.05)$.

HRs of patients in the dexmedetomidine group and the midazolam group at different time points. At T1, T2, T3 and $\mathrm{T} 4$, the HRs of patients in the dexmedetomidine group were $(84.51 \pm 9.24) \mathrm{bpm},(85.81 \pm 8.67) \mathrm{bpm},(85.21 \pm 7.86) \mathrm{bpm}$, and $(84.13 \pm 8.23) \mathrm{bpm}$, respectively, while the HRs of patients in the midazolam group were $(83.12 \pm 8.89) \mathrm{bpm},(72.79 \pm 8.65) \mathrm{bpm}$, (65.19 \pm 8.71$) \mathrm{bpm}$, and (79.26 \pm 7.22$)$ bpm, respectively (Fig. 2). The HRs at T1 in both groups were comparable, and there was no significant difference between them $(\mathrm{P}>0.05)$. However, the HRs at T2, T3 and T4 were all higher in the dexmedetomidine group than those in the midazolam group, and the differences

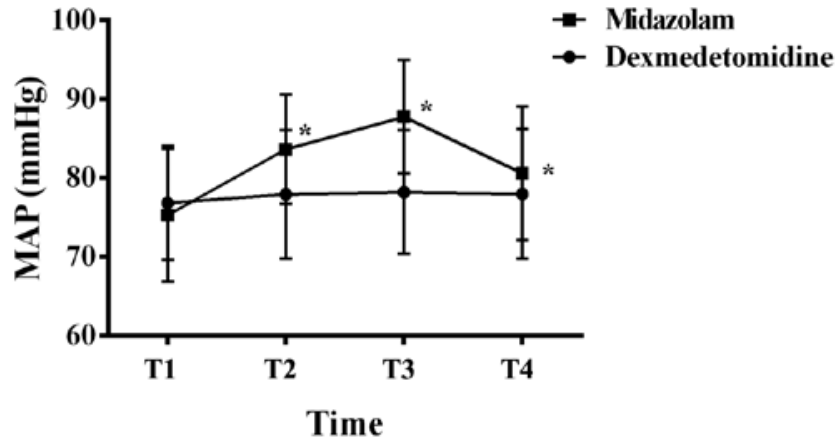

Figure 1. MAPs of patients in the dexmedetomidine group and the midazolam group at different time points. The MAPs at T1 in both groups were comparable, and there was no significant difference between them $(\mathrm{P}>0.05)$ The MAPs at T2, T3 and T4 were all lower in the dexmedetomidine group than in the midazolam group, and the differences were statistically significant $(\mathrm{P}<0.05)$. Within the dexmedetomidine group, the MAPs at each time point were comparable, and the differences were not statistically significant $(\mathrm{P}>0.05)$. Within the midazolam group, the MAPs at T2 and T3 were higher than that at the preceding time point, whereas the MAP at T4 was lower than that at the preceding time point. All the differences were statistically significant $(\mathrm{P}<0.05){ }^{*} \mathrm{P}<0.05$, a statistically significant difference from the previous time point.

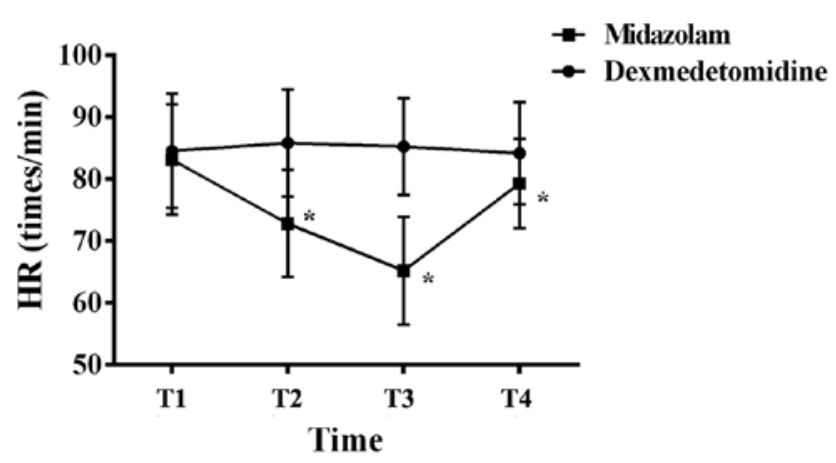

Figure 2. HRs of patients in the dexmedetomidine group and the midazolam group at different time points. The HRs at T1 in both groups were comparable, and there was no significant difference between them $(P>0.05)$. The HRs at T2, T3 and T4 were all higher in the dexmedetomidine group than in the midazolam group, and the differences were statistically significant $(\mathrm{P}<0.05)$. Within the dexmedetomidine group, the HRs at each time point were comparable, and the differences were not statistically significant ( $\mathrm{P}>0.05)$. Within the midazolam group, the HRs at T2 and T3 were lower than that at the preceding time point, whereas the HR at T4 was higher than that at the preceding time point. All the differences were statistically significant $(\mathrm{P}<0.05) .{ }^{*} \mathrm{P}<0.05$, a statistically significant difference from the previous time point.

were statistically significant $(\mathrm{P}<0.05)$. Within the dexmedetomidine group, the HRs at each time point were comparable, and the differences were not statistically significant $(\mathrm{P}>0.05)$. Within the midazolam group, the HRs at T2 and T3 were lower than that at the preceding time point, whereas the HR at T4 was higher than that at the preceding time point. All the differences were statistically significant $(\mathrm{P}<0.05)$.

Serum levels of TNF- $\alpha$ of patients in the dexmedetomidine group and the midazolam group at different time points. At $\mathrm{T} 1$, the serum levels of TNF- $\alpha$ in both groups were comparable, and the difference between them was not statistically significant $(\mathrm{P}>0.05)$. However, the serum levels of TNF- $\alpha$ at 
Table II. Serum levels of TNF- $\alpha$ of patients in the dexmedetomidine and the midazolam group at different time points (pg/ml).

\begin{tabular}{llrr}
\hline Time point & Dexmedetomidine group $(\mathrm{n}=169)$ & Midazolam group $(\mathrm{n}=174)$ & $\mathrm{t}$ \\
\hline T1 & $1.13 \pm 0.32$ & $1.17 \pm 0.51$ & 0.387 \\
T2 & $5.12 \pm 2.83^{\mathrm{a}}$ & $8.61 \pm 1.15^{\mathrm{a}}$ & $<67$ \\
T3 & $3.25 \pm 0.79^{\mathrm{a}}$ & $6.99 \pm 1.71^{\mathrm{a}}$ & 15.040 \\
T4 & $3.01 \pm 0.45^{\mathrm{a}}$ & $6.18 \pm 0.67^{\mathrm{a}}$ & 25.880 \\
F & 201.4 & 1,446 & 51.290 \\
P-value & $<0.001$ & $<0.001$ & $<0.001$ \\
\end{tabular}

${ }^{\text {a }}<<0.05$, a statistically significant difference from the previous time point.

Table III. Serum levels of IL-6 of patients in the dexmedetomidine group and the midazolam group at different time points (pg/ml).

\begin{tabular}{llrr}
\hline Time point & Dexmedetomidine group $(\mathrm{n}=169)$ & Midazolam group $(\mathrm{n}=174)$ & $\mathrm{t}$ \\
\hline T1 & $27.83 \pm 12.72$ & $28.25 \pm 11.51$ & 0.321 \\
T2 & $88.83 \pm 12.71^{\mathrm{a}}$ & $61.26 \pm 18.36^{\mathrm{a}}$ & 0.748 \\
T3 & $62.28 \pm 12.63^{\mathrm{a}}$ & $49.48 \pm 12.51^{\mathrm{a}}$ & 6.788 \\
T4 & $55.51 \pm 11.79^{\mathrm{a}}$ & $43.89 \pm 18.98^{\mathrm{a}}$ & 9.429 \\
F & 682.6 & 132.7 & 16.130 \\
P-value & $<0.001$ & $<0.001$ & $<0.001$ \\
\hline
\end{tabular}

${ }^{\mathrm{a}} \mathrm{P}<0.05$, a statistically significant difference from the previous time point.

T2, T3 and T4 were all lower in the dexmedetomidine group than those in the midazolam group, and the differences were statistically significant $(\mathrm{P}<0.05)$. Within each group, the serum levels of TNF- $\alpha$ at T2, T3 and T4 were all higher than that at $\mathrm{T} 1(\mathrm{P}<0.05)$. The level peaked at $\mathrm{T} 2$, and then decreased at $\mathrm{T} 3$ and $\mathrm{T} 4$ compared to the preceding time point $(\mathrm{P}<0.05)$. The details are listed in Table II.

Serum levels of IL-6 of patients in the dexmedetomidine group and the midazolam group at different time points. At T1, the serum levels of IL-6 in both groups were comparable, and the difference between them was not statistically significant $(\mathrm{P}>0.05)$. However, the serum levels of IL-6 at T2, T3 and $\mathrm{T} 4$ were all higher in the dexmedetomidine group than in the midazolam group, and the differences were statistically significant $(\mathrm{P}<0.05)$. Within each group, the serum levels of IL-6 at T2, T3 and T4 were all higher than that at T1 $(\mathrm{P}<0.05)$. The level peaked at $\mathrm{T} 2$, and then decreased at T3 and T4 compared to the preceding time point $(\mathrm{P}<0.05)$. The details are listed in Table III.

\section{Discussion}

Our study showed that there was no significant difference in serum TNF- $\alpha$ and IL-6 levels between the two groups at T1 $(\mathrm{P}>0.05)$. The level of serum TNF- $\alpha$ in patients with dexmedetomidine at T2, T3 and T4 was lower than that of the midazolam group, and the serum IL- 6 in patients with dexmedetomidine at T2, T3, T3 was higher than that of the midazolam group $(\mathrm{P}<0.05)$. Serum TNF- $\alpha$ and IL-6 levels in the dexmedetomidine group at T2, T3 and T4 were higher than those at $\mathrm{T} 1(\mathrm{P}<0.05) . \mathrm{T} 2$ was the peak of serum TNF- $\alpha$ and IL- 6 levels. The levels of serum TNF- $\alpha$ and IL- 6 at T3 and T4 were lower than those at the preceding time points $(\mathrm{P}<0.05)$. Serum levels of TNF- $\alpha$ and IL-6 at T2, T3 and T4 in the midazolam group were significantly higher than those at $\mathrm{T} 1(\mathrm{P}<0.05)$, and $\mathrm{T} 2$ was the peak. The levels of serum TNF- $\alpha$ and IL- 6 at T3 and T4 were significantly lower than those at the preceding time points. The results of this study are consistent with the results of Djaiani et al (16), suggesting that the choice of dexmedetomidine as an anesthetic can reduce the release of pro-inflammatory factor TNF- $\alpha$ and IL- 6 induced by TNF- $\alpha$ and macrophages.

Because midazolam is a short-acting water-soluble benzodiazepine sedative, midazolam produces sedative, hypnotic, anticonvulsant, and anxiolytic effects by altering the configuration of the GABAA receptor complex and opening the chloride channel. The effect of antegrade amnesia, combined with opioids, etomidate, propofol drugs can produce synergistic or additive effects, resulting in analgesic effect (17). However, the use of midazolam can accumulate in patients with renal failure, and some patients may also develop drug resistance; while the excessive injection dose or use may cause blood pressure drop or respiratory depression in patients with hypovolemia (18). Dexmedetomidine is a highly selective novel $\alpha 2$ adrenergic receptor agonist with a specific pharmacological effect (19). Dexmedetomidine mainly acts on the locus coeruleus of the brainstem with the most concentrated $\alpha 2$ receptor in the 
central nervous system. The locus coeruleus in the brain is mainly responsible for regulating the key parts of human sleep and awakening. After dexmedetomidine is produced, people can be awakened, but there are still anxiolytic effects; at the same time, analgesic effects are produced by the synaptic and post-synaptic membrane $\alpha 2$ receptors in the spinal cord interneurons; dexmedetomidine inhibits central sympathetic nerves, enhances vagal activity, and attenuates sympathetic nerves tension, which brings better sedative effect (20). The anti-sympathetic effects of dexmedetomidine can lower blood pressure, HR, myocardial contractility, myocardial oxygen consumption, and plasma catecholamine levels, so patients gain hemodynamic stability without significant respiratory depression (21). Dexmedetomidine limits the inflammatory response of endotoxin, thereby reduces the stress response in patients (22).

There was no significant difference in MAP between dexmedetomidine group and midazolam at $\mathrm{T} 1(\mathrm{P}>0.05)$. At T2, T3 and T4, MAP index in dexmedetomidine group was significantly lower than that in midazolam group $(\mathrm{P}<0.05)$. There was no significant change in the MAP index at each time point in the dexmedetomidine group, and the difference was not statistically significant $(\mathrm{P}>0.05)$. However, the MAP index at T2 and T3 was significantly higher in the midazolam group than that in the preceding time point, and was significantly lower at T4 $(\mathrm{P}>0.05)$. There was no significant difference in the HR between the dexmedetomidine group and the midazolam group at $\mathrm{T} 1(\mathrm{P}>0.05)$. The HR index of the dexmedetomidine group at T2, T3, T4 was higher compared with that in midazolam group, and the difference was statistically significant $(\mathrm{P}<0.05)$. There was no significant difference in $\mathrm{HR}$ in the dexmedetomidine group $(\mathrm{P}>0.05)$, but $\mathrm{HR}$ index in the midazolam group at T2, T3, T4 was significantly lower, compared with preceding time points. There was no significant change in the HR index at each time point in the dexmedetomidine group $(\mathrm{P}>0.05)$. However, the HR index at $\mathrm{T} 2$ and $\mathrm{T} 3$ in the midazolam group was significantly lower compared with that of preceding time points, while it was significantly higher at T4. It shows that dexmedetomidine has less hemodynamic fluctuations compared with midazolam, which can increase the safety of patients and stabilize the life of patients. The results of MAP and HR by Barends et al (23), when using dexmedetomidine for surgery were similar to ours, which further corroborated our experiment.

After trauma, pain and stress, the tissue would be stimulated to release a large number of inflammatory factors. In the body's reaction mechanism, macrophages, B lymphocytes and $\mathrm{T}$ lymphocytes can produce TNF- $\alpha$, which is an early inflammatory reaction that further induce cytokine cascades and induce the production of other cytokines and chemokines, stimulate the release of IL-6, causing a series of inflammatory chain reactions (24). Our study examined the changes of TNF- $\alpha$ and IL- 6 levels in the perioperative period of patients undergoing laparoscopic ovarian cancer radical surgery. Therefore, during the radical operation of ovarian cancer, the choice of anesthetic drugs is the key to stabilizing the patient's vital signs and reducing the inflammatory factors in patients.

In this experiment, due to the limited medical resources in our hospital, the number of selected subjects was small, and there may be some contingency in the results. It is not excluded that there are differences in the response of anesthesia at different ages. We intend to conduct longer-term follow-up surveys.

In summary, compared with midazolam anesthesia, the use of dexmedetomidine anesthesia can more effectively stabilize the vital signs of patients, improve safety, and reduce the release of inflammatory factors. It is more suitable for ovarian cancer laparoscopic surgery, and is worthy of clinical promotion.

\section{Acknowledgements}

Not applicable.

\section{Funding}

No funding was received.

\section{Availability of data and materials}

The datasets used and/or analyzed during the present study are available from the corresponding author on reasonable request.

\section{Authors' contributions}

ML conceived and designed this study. YY collected and analyzed the data. ML, YY and MZ performed the experiments. ML and MZ wrote the manuscript and revised it critically. All the authors read and approved the final manuscript.

\section{Ethics approval and consent to participate}

The study was approved by the Ethics Committee of the Affiliated Hospital of Qingdao University (Qingdao, China).

\section{Patient consent for publication}

Not applicable.

\section{Competing interests}

The authors declare that they have no competing interests.

\section{References}

1. Salomon-Perzyński A, Salomon-Perzyńska M, Michalski B and Skrzypulec-Plinta V: High-grade serous ovarian cancer: The clone wars. Arch Gynecol Obstet 295: 569-576, 2017.

2. Ramachandran SM, Liu LY and Perkins SH. Peristomal nodule patient with ovarian cancer. JAMA 319: 1158-1159, 2018.

3. Gallotta V, Cicero C, Conte C, Vizzielli G, Petrillo M, Fagotti A, Chiantera V, Costantini B, Scambia G and Ferrandina G: Robotic versus laparoscopic staging for early ovarian cancer: A casematched control study. J Minim Invasive Gynecol 24: 293-298, 2017.

4. Dong H, Zhang Y and Xi H: The effects of epidural anaesthesia and analgesia on natural killer cell cytotoxicity and cytokine response in patients with epithelial ovarian cancer undergoing radical resection. J Int Med Res 40: 1822-1829, 2012.

5. Schaller SJ, Alam SM, Mao J, Zhao Y, Blobner M, Greenblatt DJ and Martyn JA: Pharmacokinetics cannot explain the increased effective dose requirement for morphine and midazolam in rats during their extended administration alone or in combination. J Pharm Pharmacol 69: 82-88, 2017.

6. Armstrong DK: New therapies for ovarian cancer. J Natl Compr Canc Netw 16 (5S): 632-635, 2018 
7. Park SJ, Shin S, Kim SH, Kim HW, Kim SH, Do HY and Choi YS: Comparison of dexmedetomidine and fentanyl as an adjuvant to ropivacaine for postoperative epidural analgesia in pediatric orthopedic surgery. Yonsei Med J 58: 650-657, 2017.

8. Scibelli G, Maio L, Sasso M, Lanza A and Savoia G: Dexmedetomidine: Current role in burn ICU. Transl Med UniSa 16: 1-10, 2017.

9. Pudenz M, Roth $\mathrm{K}$ and Gerhauser C: Impact of soy isoflavones on the epigenome in cancer prevention. Nutrients 6: 4218-4272, 2014.

10. Nicula R and Costin N: Management of endometrial modifications in perimenopausal women. Clujul Med 88: 101-110, 2015.

11. Chatterjee M, Hurley LC and Tainsky MA: Paraneoplastic antigens as biomarkers for early diagnosis of ovarian cancer. Gynecol Oncol Rep 21: 37-44, 2017.

12. Felix AS, Bower JK, Pfeiffer RM, Raman SV, Cohn DE and Sherman ME: High cardiovascular disease mortality after endometrial cancer diagnosis: Results from the Surveillance, Epidemiology, and End Results (SEER) Database. Int J Cancer 140: 555-564, 2017

13. Zambirinis CP and Miller G: Cancer manipulation of host physiology: Lessons from pancreatic cncer. Trends Mol Med 23: 465-481, 2017.

14. Brand AH, DiSilvestro PA, Sehouli J and Berek JS: Cytoreductive surgery for ovarian cancer: Quality assessment. Ann Oncol 28 (Suppl 8): viii25-viii29, 2017.

15. Ghodki PS, Sardesai SP and Halikar SS: Dexmedetomidine premedication in cataract surgery under topical anaesthesia: To assess patient and surgeon satisfaction. S Afr J Anaesthesiol Analg 21: 17-21 2015.

16. Djaiani G, Silverton N, Fedorko L, Carroll J, Styra R, Rao V and Katznelson R: Dexmedetomidine versus propofol sedation reduces delirium after cardiac surgery: A randomized controlled trial. Anesthesiology 124: 362-368, 2016.
17. Balk M, Hentschke H, Rudolph U, Antkowiak B and Drexler B: Differential depression of neuronal network activity by midazolam and its main metabolite 1-hydroxymidazolam in cultured neocortical slices. Sci Rep 7: 3503-3515, 2017.

18. Yasoshima Y and Shimura T: Midazolam impairs the retrieval of conditioned taste aversion via opioidergic transmission in mice. Neurosci Lett 636: 64-69, 2017.

19. Freeman J and Buggy DJ: Modelling the effects of perioperative interventions on cancer outcome: Lessons from dexmedetomidine. Br J Anaesth 120: 15-17, 2018.

20. Li Z and Sheng L: Significance of dynamic evolution of TNF- $\alpha$, IL-6 and intestinal fatty acid-binding protein levels in neonatal necrotizing enterocolitis. Exp Ther Med 15: 1289-1292, 2018.

21. Kim Y, Lim HJ, Jang HJ, Lee S, Jung K, Lee SW, Lee SJ and Rho MC: Portulaca oleracea extracts and their active compounds ameliorate inflammatory bowel diseases in vitro and in vivo by modulating TNF- $\alpha$, IL-6 and IL- $1 \beta$ signalling. Food Res Int 106: 335-343, 2018.

22. Gupta B, Verma RK, Kumar S and Chaudhary G: Comparison of analgesic efficacy of dexmedetomidine and midazolam as adjuncts to lignocaine for intravenous regional anesthesia. Anesth Essays Res 11: 62-66, 2017.

23. Barends CR, Absalom A, van Minnen B, Vissink A and Visser A: Dexmedetomidine versus midazolam in procedural sedation. A systematic review of efficacy and safety. PLoS One 12: e0169525, 2017.

24. Field C and Goff BA: Dermatomyositis - key to diagnosing ovarian cancer, monitoring treatment and detecting recurrent disease: Case report. Gynecol Oncol Rep 23: 1-3, 2017.

(i) $($ This work is licensed under a Creative Commons Attribution-NonCommercial-NoDerivatives 4.0 International (CC BY-NC-ND 4.0) License. 